\title{
BOUNDS ON THE DECAY RATE OF DAMPED LINEAR SYSTEMS*
}

BX E. E. ZAJAC (Bell Telephone Laboratories, Murray Hill, New Jersey)

The decay rate in a damped linear dynamical system is usually of considerable interest. This rate is related to the distance from the imaginary axis of the farthest right root of the system's characteristic equation. Upper bounds for this distance which are sometimes useful are given by the following theorem: Write the characteristic equation in the form

$$
a_{0} z^{n}+\left(\begin{array}{c}
n \\
1
\end{array}\right) a_{1} z^{n-1}+\cdots+\left(\begin{array}{c}
n \\
m
\end{array}\right) a_{m} z^{n-m}+\cdots+a_{n}=0,
$$

where the $a_{m}$ are real. Assume also that

$$
a_{m}>0, \quad m=0,1, \cdots, n,
$$

as occurs in damped systems where the roots are all in the open left half-plane. Let the right-most root lie on the line $\operatorname{Re} z=-D$. Then

$$
\frac{a_{m+1}}{a_{m}} \geq D, \quad m=0,1, \cdots, n-1 .
$$

As a corollary, it follows that in a damped system $(D>0)$

$$
\frac{a_{m+k}}{a_{m}} \geq D^{k}, \quad m=0,1, \cdots, n-k .
$$

If the polynomial is normalized so that $a_{0}=1$, this in turn gives

$$
a_{m} \geq D^{m}, \quad m=0,1, \cdots, n .
$$

The bounds (2) are simultaneously attained when Eq. (1) has an $n$-fold negative real root.

Typically in a dynamical system the coefficients $a_{m}$ of the characteristic equation, Eq. (1), are polynomial functions of the system parameters. Although the coefficients are then unbounded functions of the parameters, ratios of some of the coefficients are usually bounded and by (3) allow one to obtain upper bounds on $D$. Although its proof is elementary, the theorem does not seem to occur in the automatic control literature, for example [1], nor does it appear in Marden's standard work, [2].

To prove the theorem, shift the origin to $z=x$ by setting $z=\xi+x$. This gives

$$
q_{0} \zeta^{n}+\left(\begin{array}{c}
n \\
1
\end{array}\right) q_{1}(x) \zeta^{n-1}+\cdots+\left(\begin{array}{c}
n \\
m
\end{array}\right) q_{m}(x) \zeta^{n-m}+\cdots+q_{n}(x)=0,
$$

where it is easily verified that

$$
\begin{aligned}
q_{0} & =a_{0}>0, \\
q_{1}(x) & =a_{0} x+a_{1}, \\
q_{2}(x) & =a_{0} x^{2}+2 a_{1} x+a_{2}, \\
\cdots \cdots \cdots \cdots \cdots \cdots \cdots \cdots \cdots \cdots \cdots \cdots & \\
q_{m}(x) & =\sum_{r=0}^{m}\left(\begin{array}{c}
m \\
r
\end{array}\right) a_{r} x^{m-r} .
\end{aligned}
$$

*Received April 2, 1962. 
If there are no roots on or to the right of $\zeta=0(z=x)$, then $q_{m}(x)>0$. But the $q_{m}(x)$ cannot be all positive for $x=-a_{m+1} / a_{m}$. For example, consider $m=3$, and form

$$
\begin{aligned}
\left(\frac{a_{4}}{a_{3}}\right)^{3} q_{1}\left(-\frac{a_{4}}{a_{3}}\right)+3\left(\frac{a_{4}}{a_{3}}\right)^{2} q_{2}\left(-\frac{a_{4}}{a_{3}}\right)+3\left(\frac{a_{4}}{a_{3}}\right) q_{3} & \left(-\frac{a_{4}}{a_{3}}\right) \\
= & -a_{0}\left(\frac{a_{4}}{a_{3}}\right)^{4}+4 a_{1}\left(\frac{a_{4}}{a_{3}}\right)^{3}-6 a_{2}\left(\frac{a_{4}}{a_{3}}\right)^{2}+3 a_{3}\left(\frac{a_{4}}{a_{3}}\right) \\
= & -q_{4}\left(-\frac{a_{4}}{a_{3}}\right),
\end{aligned}
$$

since $3 a_{3}\left(a_{4} / a_{3}\right)=4 a_{3}\left(a_{4} / a_{3}\right)-a_{4}$. Hence, positive $q_{1}(x), q_{2}(x), q_{3}(x)$ at $x=-a_{4} / a_{3}$ imply that $q_{4}(x)$ is negative. Therefore at least one root must lie on or to the right of $z=-a_{4} / a_{3}$. The generalization to $z=-a_{m+1} / a_{m}$ follows in the same way. First form

$$
Q=\sum_{r=1}^{m}\left(\begin{array}{c}
m \\
r-1
\end{array}\right)\left(\frac{a_{m+1}}{a_{m}}\right)^{m+1-r} q_{r}\left(-\frac{a_{m+1}}{a_{m}}\right) .
$$

Then by use of the identity:

$$
\sum_{\rho=s}^{n}(-1)^{\rho-s}\left(\begin{array}{c}
n \\
\rho-1
\end{array}\right)\left(\begin{array}{l}
\rho \\
s
\end{array}\right)= \begin{cases}(-1)^{n-s}\left(\begin{array}{c}
n+1 \\
s
\end{array}\right), & s \neq n \\
n, & s=n\end{cases}
$$

it follows that $Q=-q_{m+1}\left(-a_{m+1} / a_{m}\right)$. Hence the $q_{m}(x)$ cannot all be positive for $x=$ $-a_{m+1} / a_{m}$ and Inequality (2) is established. Identity (5) can be obtained by expanding the right side of $(1-x) x^{n}=[1-(1-x)]^{n}(1-x)$ in a power series in $x$ and comparing coefficients of like powers.

If the polynomial, Eq. (1), is of alternating sign, then by replacing $z$ by $-z$ one obtains from (2) a bound on the distance into the right half-plane of the left-most root.

\section{REFerences}

1. E. M. Grabbe, S. Ramo, D. E. Wooldridge, Handbook of automation, computation, and control, Wiley, New York, 1958, vol. 1, chap. 21

2. M. Marden, Geometry of the zeros of a polynomial, Amer. Math. Soc., 1949 